\title{
How can we associate an economic evaluation with a clinical trial?
}

\section{Mariana Minatel BRAGA ${ }^{(a)}$ iD Gabriela Manco MACHADO(a) (ID Elizabeth Souza ROCHA ${ }^{(a)}$ iD Maria Eduarda VIGANÓ(a) (ID Laura Regina Antunes PONTES(a)}

Daniela Prócida RAGGIO(a)

(a) Universidade de São Paulo - USP, School of Dentistry, Department of Paedriatric Dentistry, São Paulo, SP, Brazil.
Declaration of Interests: The authors certify that they have no commercial or associative interest that represents a conflict of interest in connection with the manuscript.

\section{Corresponding Author:}

Mariana Minatel Braga

E-mail:mmbraga@usp.br

ht1ps://doi.org/10.1590/1807-3107bor-2020.vol34.0076

Submitted: September 4, 2019

Accepted for publication: September 22, 2019 Last revision: June 13, 2020
Abstract: Economic evaluations in Dentistry have been increasing in recent years. They are a relevant contribution if an economic issue exists. Knowing if a new intervention is an efficient way of allocating available (and scarce) resources (the concept of opportunity costs), a well-designed economic evaluation may be helpful. One option is to conduct a trial-based economic analysis, which extracts a considerable board of information from a trial. This approach produces a more controlled result since many sources of variations might be reduced. On the other hand, some aspects could not be predicted directly from the trial or even extrapolated. Thus, combining model-based analysis may be an idea. In this paper, we intended to discuss important aspects to be considered by researchers in further economic evaluations. This paper will be systematically divided into sessions related to the study design as time horizon and perspective, health effects, costs, and data analysis. In the end, we expect the reader could be able to plan a trial-based economic evaluation, which should be a careful, meticulous, quite laborious and especially transparent process.

Keywords: Cost-Benefit Analysis; Clinical Trials as Topic; Dentistry.

\section{Introduction}

On the one hand, the evidence-based practice advocates such type of practice will opt for most efficacious interventions to maximize the quantity and quality of life of patients (i.e., optimize the effect of a tested approach). ${ }^{1}$ On the other hand, we cannot assure the evidence-based practice will necessarily represent the best way to allocate financial resources. Some believe evidence-based practices are a way to cut the costs of health care ${ }^{1}$. In general, others agree that such type of practice tends to mostly increase costs of health care instead of reducing it. ${ }^{1}$ Solving this duality points to the actual relevance of economic evaluations related to health care. Therefore, economic evaluations are a way of merging two conceptually different worlds: clinicians' (effectiveness world) and economists' world (efficiency world).

Efficiency is essentially related to some economic principles, not generally usual to health care providers, such as scarcity, for example. Health is a scarce resource, as well as financial resources. Thus, making choices must involve decision-making about how the available resources could be shared. ${ }^{2}$ Another important principle is the opportunity costs. 
As resources are scarce, when a new evidence-based strategy is available and needs to be implemented, resources previously allocated to another purpose should be reallocated and redirected to this new strategy. That is why efficient resource allocation must be required. In other words, the benefits of a certain choice should exceed the benefits of any alternative. ${ }^{2}$

When a new effective evidence-based strategy is available, its implementation cost should be estimated. Then, besides the effect that the strategy can result, providers or health systems should evaluate how the resources should be dimensioned to make such implementation possible and if it is an efficient way to allocate resources. However, as clinicians or health care providers, we usually do not think about that, leaving it more to the economists' field.

The attempt to merge these two different worlds is not always a simple task. While one argues, "... health economists, while seeking to colonize the clinical mind may have lost their disciplinary head...", ${ }^{3}$ another contributes "Economics provides useful (but not simple) methods to help sort out resource allocation to optimize benefits...." ${ }^{4}$ Based on the economic principles exposed above, which should guide economic evaluations, we invite the readers to reflect if the papers published and related to the health economic field are looking for the most efficient way of allocating resources. In other words, are researchers motivated to investigate if a new treatment is a better way of allocating resources than a standard or conventional option? Or was the economic evaluation automatically included in the trial? Are these studies satisfying the economic principles that should guide economic evaluations? Are they contributing to including or not the evidencebased practice in real life? We hope that, by reading this paper, readers may judge the efficient ways of allocating resources for health care. Besides, when planning economic evaluations that they could be able to contribute to this knowledge for clinicians, providers, and payers.

\section{Planning and Implementation}

To assert or answer about efficient resource allocation considering a health care strategy (e.g., some dental treatment or diagnostic approach), economic evaluations should be designed combining simultaneously two different outcomes: the health effects, health statuses, or consequences related to the tested strategies (outputs) along with costs (inputs). Evaluations considering only costs or only effects are defined as partial evaluations. ${ }^{5}$ They are not sufficient to provide evidence for decision-making since they do not permit the comparison of inputs and outputs related to the tested alternatives.

Economic evaluations may be divided into trial-based and model-based evaluations. Both strategies present the pros and cons. They should be weighed considering the answer you expect and also limitations related to each methodology. In this paper, we will discuss the trial-based strategy. In this case, the trial could be designed specifically for economic evaluation, but it is not the most common situation. Very often, in a trial designed to evaluate other primary endpoints, if the question about efficiency in resource allocation is valid, the piggyback approach is the strategy used to implement trial-based economic evaluations.

As trial-based economic analysis extracts a considerable board of information from a trial, a more controlled result could be expected, and many sources of variations might be limited. On the other hand, if a single trial was used to base an economic evaluation on, its power of generalizability and extrapolation is known to be reduced, and one should be careful when making decisions based only on its results. ${ }^{6}$ As the evidence produced by a single trial must be placed in a broader framework for decision analysis, sometimes studies provide a combination of trial and model-based strategies to make a broader economic analysis and inform the decision-making process more accurately. Another possibility would be designing a more pragmatic clinical trial, improving the power of finding generalizability.

To improve the comprehension of those aspects that should be considered when planning a trial-based economic evaluation, we will systematically divide the session into topics: time horizon and perspective, health effects, costs, and data analysis.

\section{Time horizon and perspective}

When designing an economic evaluation, some definitions are supposed to be done a priori, as the 
perspective and the time horizon to be used. Perspective could be compared to the lens you decide to use to investigate the situation. It could be defined as patients', providers', payers', health care systems', or societal (the latter combining all actors and circumstances involved in the process) perspectives. While the patients' perspective is the most proximal one, societal is the most distal perspective (Figure 1a). The perspective determines both the type of effects and costs that should be considered in the analysis. It is reasonable that more distal the perspective, more complex the analysis.

Another aspect to consider is the time horizon. If the perspective could be compared to the format of a lens, allowing us to see a broader or more restricted field, the time horizon would be how far these lenses could reach (Figure 1). The time horizon is strongly related to the effects or health statuses that are supposed to be considered in the evaluation (Figure 1b). It is also related to the motivation for the evaluation. Once a perspective and time horizon have been defined, the range of resources can be identified. The aspects mentioned above could interfere significantly with the findings of such evaluation and may change the idea disseminated at the end.

\section{Health effects}

Outcomes pertinent to economic evaluations should be, first, chosen and then collected. When planning a trial-based economic evaluation, it is important to consider which outcomes could be measured and used as effects in this analysis. Firstly, this decision should be linked to feasible (available) endpoints to be collected (or even already collected) in the trial. Secondly, these endpoints should also be linked to the analysis that the researcher intends to do. Depending on the outcomes defined, a different type of analysis may be necessary (Figure 2).

Different types of economic evaluations are known, e.g., cost-effectiveness analysis (CEA), cost-utility analysis (CUA), cost-benefit analysis (CBA), among others (Figure 2). It is crucial to weigh the pros and cons related to each analysis. The health effect set as the outcome should be determined considering the economic question one wants to answer. In the following paragraphs, we will discuss some aspects related to different types of analyses and related endpoints. To facilitate the process, we draw the box below to summarize these relevant points (Figure 2).

The most frequent type of economic analysis in Dentistry is CEA. In this analysis, a health effect is considered as the output (Figure 2). This effect is usually related to the condition and area studied in Dentistry. The usefulness of the economic evaluation for guiding future decisions depends on the robustness of the outcome (endpoint).Contrariwise, some economic analyses have still been based on intermediary or surrogate outcomes, ${ }^{7}$ even given their limited power of extrapolation to decisions directed to the society. The use of appropriately validated surrogate endpoints should be considered carefully, and researchers should judge if they represent a way of speeding up access to an important asset for patients in healthcare contexts. ${ }^{8}$

Although CEA is a common option in Dentistry and it guarantees a good answer regarding the intervention alternatives by themselves, such type of strategy usually brings a professional-centered or a problem-centered outcomes, which not always have the same weigh as when judged by users, patients. Moreover, when resource allocation is the deal, cost-effectiveness analysis may present some limitations. The comparison between alternatives for intervening in different conditions/diseases may not be a simple task. This problem can be easily understood if we suppose an example. As a manager in a health system, you have some extra dollars in your budget to spend on a new dental strategy to be implemented in a municipality. Then, you receive the information that a therapy A, to prevent caries, costs an additional 1,000 dollars per cavity avoided compared to the standard care for caries prevention.

On the other hand, therapy B, to treat periodontal disease, costs the same, but per reduction in periodontal pockets. What should I choose as a health system manager? What is the most efficient way to allocate the extra budget? This question is certainly difficult to answer because you are working with different diseases and different outcomes, making a direct comparison between them very tricky.

In this sense, CUA may be a useful option. Economic evaluations like that use a generic measure for health gain. One of the most used measures is Quality-Adjusted Life-Years (QALY). To obtain 
A
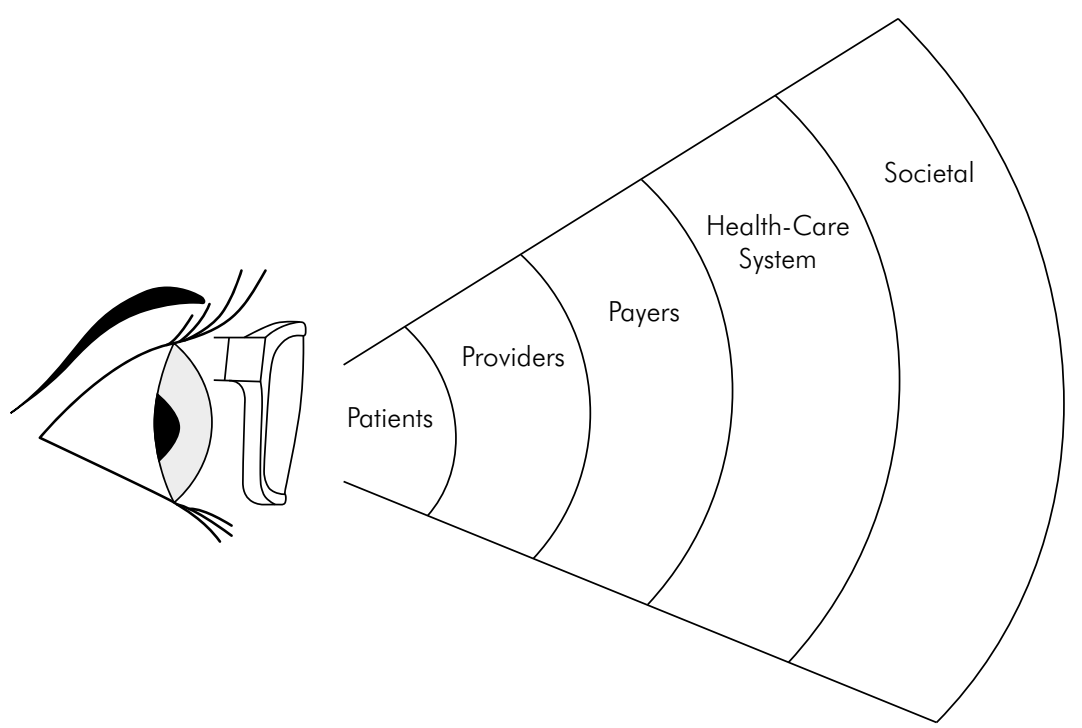

B

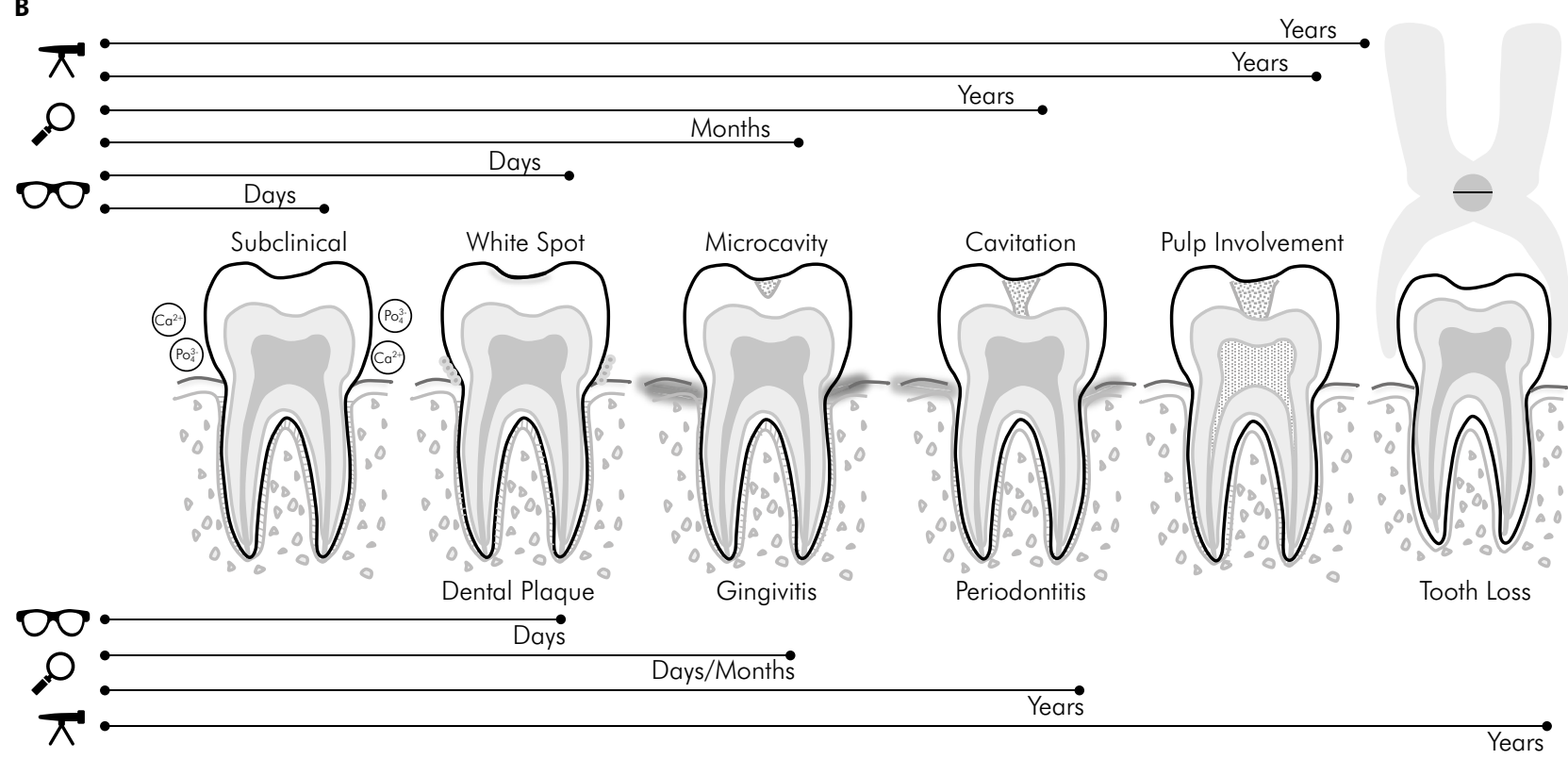

Figure 1. (a) Different perspectives of an economic evaluation. (b) Effect of time-horizon on possible health effects to be collected.

this general measure of health effect, patients and/or society must qualify the health status or conditions related to a certain disease according to their preference. ${ }^{9}$ These weights attributed to each condition are known as utility, which varies from 0 (dead or worst health condition) to 1 (healthy). When different interventions are tested, and the health status or the condition are measured after that, researchers can calculate the QALY considering the utility value of that health status and the time spent in it (Figure 2). Usually, results are expressed as cost per health years gained or cost per QALY gained by choosing one strategy instead of the other one (Figure 2). Considering the example above about the health system manager, you could assess, using the cost-utility analysis, how much we would spend per QALY to implement the new strategy for caries prevention or periodontal pocket reduction and, therefore, decide about the most advantageous way for allocating the resources as mentioned above.

On the other hand, the use of QALYs in CUA is not fully accepted by health economists. ${ }^{10}$ Depending on 


\begin{tabular}{|c|c|c|c|c|c|}
\hline Tool & 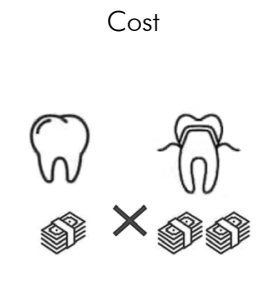 & $\begin{array}{l}3 \pi \\
\frac{1}{4}+5\end{array}$ & 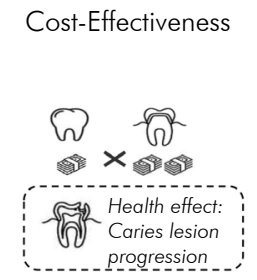 & 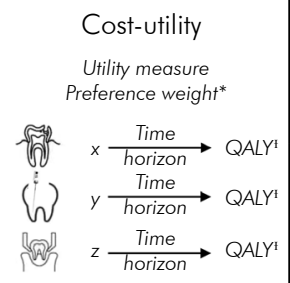 & 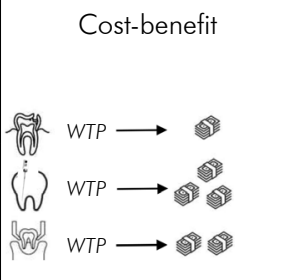 \\
\hline Measured Effect & Monetary & Monetary & Health Effect & $\begin{array}{c}\text { Health gain or } \\
\text { health-related quality } \\
\text { of life }\end{array}$ & $\begin{array}{l}\text { Willingness to pay } \\
\text { (WTP) }\end{array}$ \\
\hline Advantages & $\begin{array}{l}\text { Non-complex tool } \\
\text { Helpful with decisions } \\
\text { based only on } \\
\text { monetary units }\end{array}$ & $\begin{array}{l}\text { Non-complex tool } \\
\text { (restricted to a cost } \\
\text { comparison, when } \\
\text { effects are equivalent) }\end{array}$ & $\begin{array}{l}\text { Dependent on } \\
\text { endpoints already } \\
\text { collected in trials } \\
\text { Endpoints easier } \\
\text { to be collected, } \\
\text { measured }\end{array}$ & $\begin{array}{l}\text { Consider individuals or } \\
\text { society preferences } \\
\text { Comparability } \\
\text { between effects of } \\
\text { different natures }\end{array}$ & $\begin{array}{l}\text { Translate effects into a } \\
\text { monetary value } \\
\text { Most reliable and } \\
\text { direct tool for } \\
\text { decision-making. }\end{array}$ \\
\hline Disadvantages & $\begin{array}{c}\text { No relationship } \\
\text { between inputs and } \\
\text { outputs } \\
\text { Insufficient for } \\
\text { decision making }\end{array}$ & $\begin{array}{l}\text { Difficulty of having } \\
\text { exactly equal effects } \\
\text { in real-life. (leading to } \\
\text { misusing of this tool) }\end{array}$ & $\begin{array}{l}\text { Impossibility of } \\
\text { comparing among } \\
\text { different effects } \\
\text { and diseases } \\
\text { Non-inclusion of } \\
\text { patients' preferences } \\
\text { Difficulty in } \\
\text { guiding resources } \\
\text { reallocation in a } \\
\text { broader area }\end{array}$ & $\begin{array}{l}\text { Dependence on } \\
\text { knowing the utility } \\
\text { value or individual } \\
\text { preference to weigh } \\
\text { the effects } \\
\text { Concerns about } \\
\text { measuring and } \\
\text { quantifying preferences } \\
\text { Low morbidity of } \\
\text { oral-related conditions } \\
\text { vs. use of generic } \\
\text { preference measures }\end{array}$ & $\begin{array}{l}\text { Dependence on } \\
\text { knowing local } \\
\text { WTP related to the } \\
\text { studied effect } \\
\text { Extra }\end{array}$ \\
\hline
\end{tabular}

*Some value between 0 and 1, 'Quality-Adjusted Life Years

Figure 2. Different types of economic evaluations and different effects supposed to be measured when conducting them.

different conditions and scenarios, we doubt about QALYs comparability. ${ }^{11}$ Due to that, some alternatives have been proposed to QALY, for example, the Health Years Equivalent (HYE). ${ }^{12} \mathrm{HYE}$ permits to acknowledge the time aspect in the patients' health, avoiding, for instance, a "timeless" weight attributed to utility values when using QALY. ${ }^{13}$

Although CUAs are the most published economic analyses in health sciences nowadays, this strategy has been more widely used in Dentistry recently. Some authors have investigated the patients' preferences and converted them into QALY ${ }^{14}$ or assessing the HYEs. ${ }^{15}$ HYEs and QALYs produced different scores for CUAs, even when chronic statuses related to dental treatment were considered. ${ }^{15}$

Other authors have created an adapted QALY measure to be used in Dentistry, the Quality-Adjusted Tooth-Year (QATY) ${ }^{16,17}$ For the QATY, the tooth is considered as the health unit (instead of the patient). In this case, the tooth loss would be classified as the worst score (utility score $=0$ ). Finally, utility estimates based on the oral-health quality of life scores have been proposed more recently as a manner to incorporate quality of life outcomes as patients' preference in CUA..$^{17,18}$ Despite not ideal, these purposes may offer (even temporarily) utility values to be used in economic evaluations, guaranteeing approximate comparability of dental or oral injuries to other ones. On the other hand, most dental problems are not related to high morbidity conditions. That is why most questionnaires usually used to estimate utility values are not capable of discriminating the impact and the value of a dental problem..${ }^{19}$ Oral-health related quality of life questionnaires could be more helpful in this sense. ${ }^{18}$

Another manner of comparing interventions in terms of decision-making is CBA. Its advantage is 
the valuation of consequences related to the tested interventions using monetary units. Its use for health is still modest (even more in Dentistry). The monetary valuation of different effects of interventions allows a direct link to the decision-making process. For that, individuals/society should express their potential willingness-to-pay (WTP) for health benefits or effects (Figure 1). When WTP values are not available, parallel well-designed studies ${ }^{20}$ are supposed to be planned to obtain these values and permit performing such type of analysis. ${ }^{21}$ Therefore, the CBA may produce an interesting answer for the economic question, but it may demand an additional effort. The WTP should be calculated depending on the perspective set for economic evaluation. In Dentistry, one example of using WTP is related to the use of anesthetic gel to be used in periodontal sessions. ${ }^{22}$

When planning a trial to be associated with economic evaluation, appropriate endpoints (health effects) should be chosen. If necessary, separate surveys should be undertaken to collect extra information supposed to be required in them. Finally, the economic analysis plan must contemplate the strategy intended to be used in the evaluations. The different strategies for health economic analyses are tools to assess what you need to answer the economic question.

\section{Costs}

Differently from the health effects (usually collected in efficacy or effectiveness trials), to nest an economic evaluation in a trial, it is also important to collect costs related to health care strategies or interventions. In this sense, two main questions may be raised: a) Which costs should be considered? b) How should these costs be measured or estimated? This section aims to clarify these points.

It is important to mention that resources are not just financial. An in-depth analysis is necessary to identify all resources related to the perspective defined (Figure 3a). A certain item could have a value from a perspective but not from another (Figure 3b). For example, under a societal point-of-view, it is expected to include not only costs to provide the treatment but also costs derived from receiving the treatment, e.g., loss of productivity, time spent, and transportation expenses, among others (Figure $3 b$ ).
You can consider as a direct resource, the hour/cost of a professional or a team/staff, materials, equipment (acquisition, maintenance, and depreciation), power supplies, and accommodation. The indirect cost may be divided into the cost associated with the procedure or associated with the patient. The effort that your patient must do to receive that service, for example, productivity loss, commuting costs, among others are considered indirect costs. The indirect cost associated with the procedure may be the need for other services: specialized services, hospital, and prosthetic laboratory, for example (Figure 3a). At this stage, it is possible and interesting to organize the list of resources on a board, e.g., a decision tree or a flowchart, to understand the invested resources.

The following step consists in defining how these resources will be valued. Firstly, in trial planning, this step is crucial to determine which cost-related variables should be measured along with the trial and when data collection should be done. Different formats are acceptable since the information could be collected accurately and efficiently. Then, a value should be attributed to each one of the resources (types of cost defined a priori). For some resources, e.g., materials, the quantities may be measured, and the market price collected. In other cases, e.g., non-market resources, prices do not always reflect the value of resources. Then, the valuation should consider credible references related to the scenario, perspective, and time horizon chosen. That is why, even at a protocol phase, a detailed and consistent description of the type of costs and strategies to their valuation are required. As an example, in their protocol, Ladewig et al. ${ }^{23}$ brought a detailed description of cost valuation performed alongside a trial comparing different strategies for the restoration of primary molars.

Possible sources to collect useful information when valuing resources in economic evaluations are governmental or official local databases, scientific literature, or pilot study data. For example, to evaluate the professional cost, one can consider the local minimum wage for the specific professional class as a local monetary scale. ${ }^{23}$ Besides, if this information does not exist, information about the useful clinical hour value might be collected among a group of 
A

DIRECT

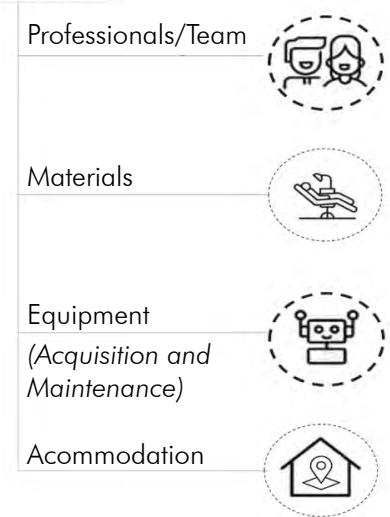

$\cos T$

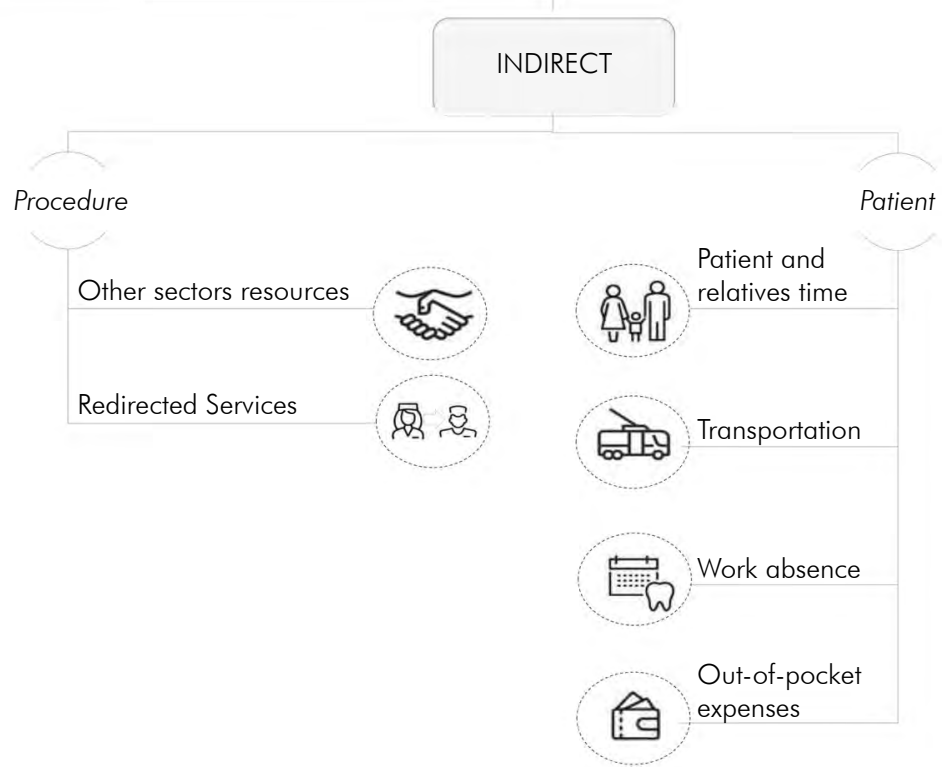

B

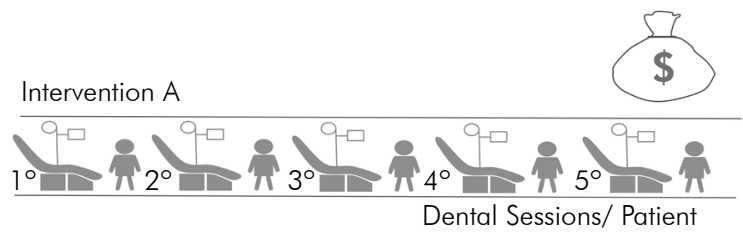

Intervention B

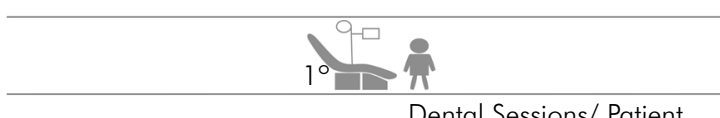

Dental Sessions/ Patient
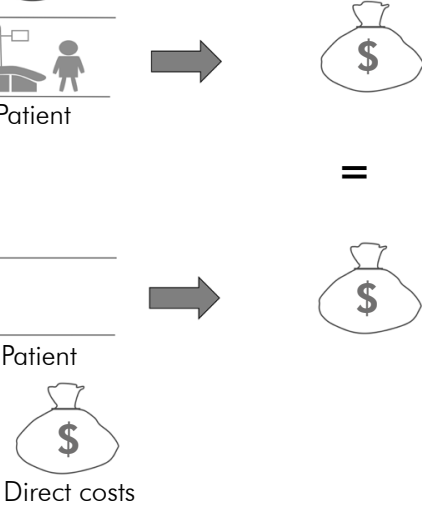

Payer's perspective (e.g. Public Health System)
How much does the treatment actually cost?

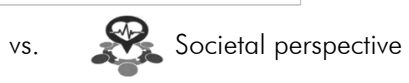

al, staff, equipment etc)

Figure 3. (a) Direct and Indirect costs related to the tested interventions. (b) Simulation of economic evaluation and costing (cost valuation) using different perspectives.

professionals, and an average cost could be considered. The perspective would also be important to determine how the cost would be valued.

One advantage of collecting data for economic evaluation alongside a trial is the possibility of registering the real costs related to the tested strategies more accurately. Some variables could be measured directly (e.g., materials) and others are time- (e.g., accommodation, equipment depreciation, professional costs) or session-related (e.g., transportation cost, absence to work). These features will guide data collection related to each variable of interest. On the other hand, necessary data may not be available, and estimation or imputation may be good strategies to 
avoid missing data. Missing data are not desirable in trial-based economic evaluations ${ }^{24}$ since they can mask important aspects of tested interventions.

In a hypothetic trial to test different strategies for caries prevention, one strategy requires annual visits, while the other demands biannual visits. However, a significant number of patients (that are supposed to) did not comply with the follow-up visits to be supervised every 6 months. If you did not consider the cost of these biannual visits, you would underestimate the cost of the biannual strategy. Maybe, the system did not spend money to actually "treat" this non-compliant patient, but the entire structure to maintain the program would be ready. In these cases, among other possibilities, you could input data about costs from other follow-up visits or based on similar patients in the same follow-up visit. Similar strategies could be adopted in cases of trials in which not all variables could be collected due to implementation issues (e.g., pragmatic trials) or design particularities (e.g., placebo-controlled trials in which the time spent only for active intervention is not available or trials in which after the event is registered, patients are redirected, and the costs cannot be measured directly).

Figure 4 shows an example of a trial-based economic evaluation conducted by our research group comparing different interventions for caries lesions control using glass ionomer cement. We simulated a circumstance in which the actual costs of the procedure were not available in a long-term analysis, but the events are known. Considering the baseline costs of both interventions and the need for re-intervention during 1 year (events), a group of experts and clinicians estimated the costs of re-interventions for each group..$^{25}$ To validate our estimation method, we measured and compared the real costs after 1 year (including re-interventions) to the estimated costs. We observed they were quite similar. We believe estimation, as proposed, could be a useful strategy, but it should be an incredibly careful and transparent process.

\section{Analysis}

Economic analyses demand a data analysis plan. This plan should be established a priori, preferably

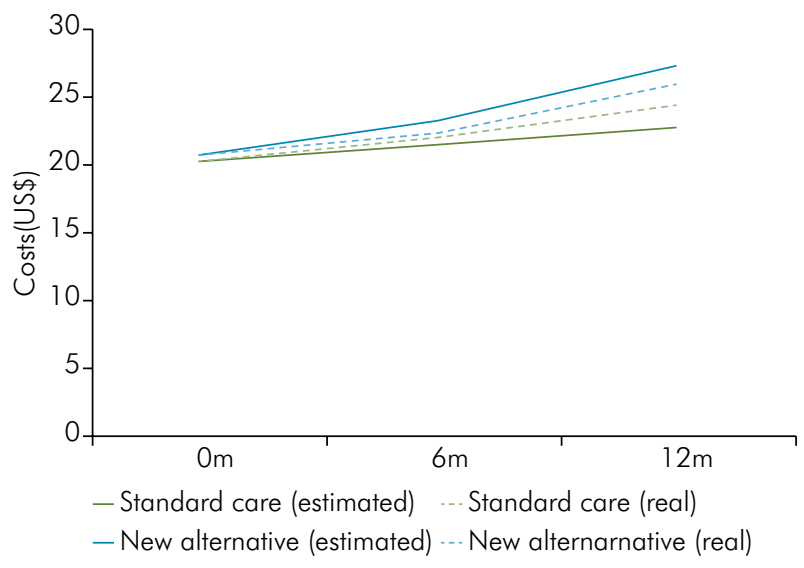

Figure 4. Comparison of estimated (simulating the absence of long-term cost data) and real cost values in a trial-based economic evaluation.

when the data is still blinded. It is recommended, as the best practice, that the analysis plan is published before completing the trial. ${ }^{24}$

In the economic analysis plan, researchers should predict which kind of statistical analyses will be conducted after data collection, including the strategies used (e.g., type of strategy), data treatment (e.g., missing data), time preference (e.g., discounting), exploration of other possible scenarios (sensitivity analyses) and also, uncertainty. Additionally, subgroup analyses should be planned, if necessary.

Even extracting data from a trial, data should be managed to allow plotting a cost-effectiveness plane (Figure 6). This plane is a graphic representation of the economic evaluation, since the outcomes explored are plotted in the axis (X: effects, Y: costs). Usually, the incremental approach is used. Then, the combination of incremental costs over incremental effects (the extra amount we are paying to have an extra effect) may be analyzed.

The position of this combination on the four quadrants in the plane may lead to the interpretation of what happens when the alternative treatment is applied instead of the standard one (Figure 6). The best option would be the southeast quadrant (most effective, less costly), but, in the northeast quadrant, the alternative would also be acceptable depending on decision rules (e.g., WHO, NICE thresholds ${ }^{26,27}$ ) or also other possible decision-making strategies ${ }^{28}$ ). In this plane, it is also explored the uncertainty related to the 


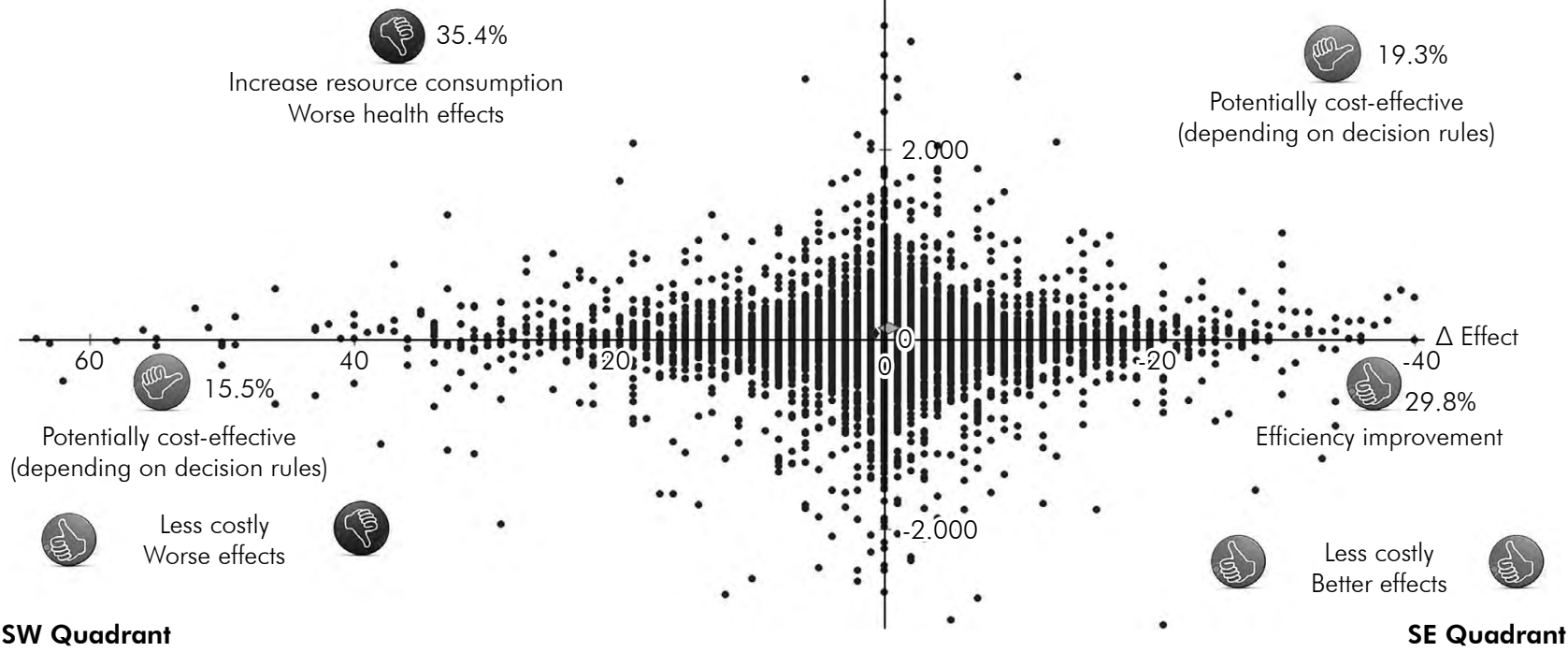

Figure 5. Cost-effectiveness plane - simulated points to explore uncertainties around the central values of incremental costs (delta cost) and effects (delta effect). Percentage values in the quadrant represent the probability of the new intervention is in that determined area of the plane (Quadrants: NE-North-East; NW: North-West; SE: South-East; SW: South-West).
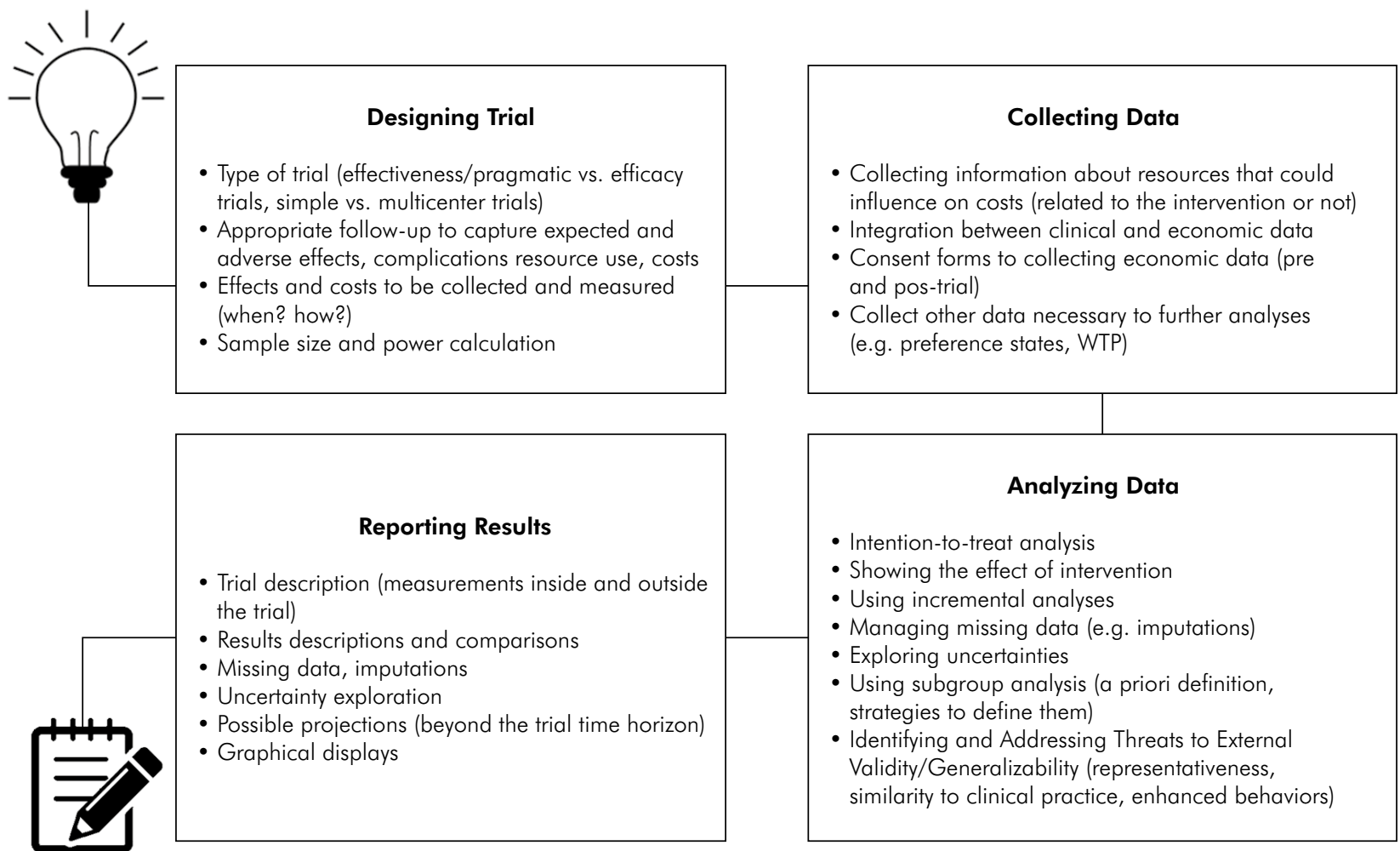

Figure 6. Schematic process summarizing important points from the idea to the protocol, according to ISPOR Good Research Practices Taskforce for economic evaluation. 
decision. In Figure 6, uncertainty can be evidenced by plots obtained from a simulation $(x 10,000)$ based on data collected from a trial. ${ }^{29}$ Depending on how these points are distributed among the quadrants, we can estimate the uncertainty surrounding the decision. For example, in this case, the decision is extremely uncertain (quite similar proportions in each quadrant), presenting a slight trend to be more concentrated in the northwest quadrant (less effective, more costly). Exploring the uncertainty guides decisions related to the adoption of the new alternatives (usually guided by a lower level of uncertainty) and to assess the need of further evidence and the potential value of acquiring additional evidence to guide decisions in the future.

\section{Final considerations}

Writing a protocol for a trial in which you intend to attach an economic evaluation should be a careful, meticulous, quite laborious and especially transparent process. Figure 6 summarizes the main points an investigator should consider when conducting and reporting a trial-based economic evaluation according to the ISPOR Good Research Practices Taskforce. ${ }^{24}$

\section{References}

1. Sackett DL, Rosenberg WM, Gray JA, Haynes RB, Richardson WS. Evidence based medicine: what it is and what it isn't. BMJ. 1996 Jan;312(7023):71-2. https://doi.org/10.1136/bmi.312.7023.71

2. Gafni A. Economic evaluation of health care interventions: an economist's perspective. ACP J Club. 1996 Mar-Apr;124(2):A12-4.

3. Maynard A, Sheldon TA. Health economics: has it fulfilled its promise? In: Maynard AC, editor. Non-random reflection on health services research. London: BMJ Press; 1977. p. 149-65.

4. Gafni A. Clouded Thinking. In: Montori VM, editor. Evidence-based endocrinology contemporary endocrinology. Totowa: Humana Press; 2006. p. 225-39. https://doi.org/10.1007/978-1-59745-008-9_15

5. Drummond MF, Sculpher MJ, Claxton K, Stoddart GL, Torrance GW. Methods for the economic evaluation of health care programmes. 4th ed. Oxford: Oxford University Press; 2015.

6. Sculpher MJ, Claxton K, Drummond M, McCabe C. Whither trial-based economic evaluation for health care decision making? Health Econ. 2006 Jul;15(7):677-87. https://doi.org/10.1002/hec.1093

7. Velasco Garrido M, Mangiapane S. Surrogate outcomes in health technology assessment: an international comparison. Int J Technol Assess Health Care. 2009 Jul;25(3):315-22. https://doi.org/10.1017/S0266462309990213

8. Ciani O, Buyse M, Drummond M, Rasi G, Saad ED, Taylor RS. Use of surrogate end points in healthcare policy: a proposal for adoption of a validation framework. Nat Rev Drug Discov. 2016 Jul;15(7):516. https://doi.org/10.1038/nrd.2016.81

9. Matthews DC, Gafni A, Birch S. Preference based measurements in dentistry: a review of the literature and recommendations for research. Community Dent Health. 1999 Mar;16(1):5-11.

10. Mehrez A, Gafni A. The healthy-years equivalents: how to measure them using the standard gamble approach. Med Decis Making. 1991 Apr-Jun;11(2):140-6. https://doi.org/10.1177/0272989X9101100212

11. Gafni A. Proper preference-based outcome measures in economic evaluations of pharmaceutical interventions. Med Care. 1996 Dec;34(12 Suppl):DS48-58.

12. Mehrez A, Gafni A. Quality-adjusted life years, utility theory, and healthy-years equivalents. Med Decis Making. 1989 Apr-Jun;9(2):142-9. https://doi.org/10.1177/0272989X8900900209

13. Gafni A. The standard gamble method: what is being measured and how it is interpreted. Health Serv Res. 1994 Jun;29(2):207-24.

14. Cunningham SJ, Sculpher M, Sassi F, Manca A. A cost-utility analysis of patients undergoing orthognathic treatment for the management of dentofacial disharmony. Br J Oral Maxillofac Surg. 2003 Feb;41(1):32-5. https://doi.org/10.1016/S0266-4356(02)00285-1

15. Birch S, Gafni A, Markham B, Marriott M, Lewis D, Main P. Health years equivalents as a measurement of preferences for dental interventions. Community Dent Health. 1998 Dec;15(4):233-42.

16. Zitzmann NU, Krastl G, Weiger R, Kühl S, Sendi P. Cost-effectiveness of anterior implants versus fixed dental prostheses. J Dent Res. 2013 Dec;92(12 Suppl):183S-8S. https://doi.org/10.1177/0022034513504927

17. Mohd-Dom T. Quality-adjusted tooth years (QATY) as an outcome measure of periodontal treatment. 7th Postgraduate Forum on Health Systems and Policies; Thailand: BMC Public Health; 2014;14(Suppl 1):P10.

18. Braga MM. R.D. F, Floriano I, S. RE, Raggio DP, Mendes FM. Proposing a utility scale related to dental caries in children based on ECOHISS scores. 66th ORCA Congress; Cartagena: Caries Research; 2019. p. 368. 
19. Foster Page LA, Beckett DM, Cameron CM, Thomson WM. Can the Child Health Utility 9D measure be useful in oral health research? Int J Paediatr Dent. 2015 Sep;25(5):349-57. https://doi.org/10.1111/ipd.12177

20. O'Brien B, Gafni A. When do the "dollars" make sense? Toward a conceptual framework for contingent valuation studies in health care. Med Decis Making. 1996 Jul-Sep;16(3):288-99. https://doi.org/10.1177/0272989X9601600314

21. Matthews D, Rocchi A, Wang EC, Gafni A. Use of an interactive tool to assess patients' willingness-to-pay. J Biomed Inform. 2001 Oct;34(5):311-20. https://doi.org/10.1006/jbin.2002.1032

22. Matthews DC, Birch S, Gafni A, DiCenso A. Willingness to pay for periodontal therapy: development and testing of an instrument. J Public Health Dent. 1999;59(1):44-51. https://doi.org/10.1111/j.1752-7325.1999.tb03234.x

23. Ladewig NM, Sahiara CS, Yoshioka L, Olegário IC, Floriano I, Tedesco TK, et al. Efficacy of conventional treatment with composite resin and atraumatic restorative treatment in posterior primary teeth: study protocol for a randomised controlled trial. BMJ Open. 2017 Jul;7(7):e015542. https://doi.org/10.1136/bmjopen-2016-015542

24. Ramsey SD, Willke RJ, Glick H, Reed SD, Augustovski F, Jonsson B, et al. Cost-effectiveness analysis alongside clinical trials II-An ISPOR Good Research Practices Task Force report. Value Health. 2015 Mar;18(2):161-72. https://doi.org/10.1016/i.jval.2015.02.001

25. Rocha ES, Gomes RA, Floriano I, Tedesco TK, Raggio DP, Imparato JC, et al. Análise econômica considerando necessidades de reintervenção de dois tratamentos com CIV em molares decíduos com lesões de cárie moderadas. 35th SBPqO Annual Meeting; 2018; Campinas, SP, Brazil. Braz Oral Res. 2018;32(supl 2):42.

26. Hutubessy R, Chisholm D, Edejer TT. Generalized cost-effectiveness analysis for national-level priority-setting in the health sector. Cost Eff Resour Alloc. 2003 Dec;1(1):8. https://doi.org/10.1186/1478-7547-1-8

27. Appleby J. Data briefing. The NICE threshold. Health Serv J. 2007 Sep;117(6074):21.

28. Sendi P, Al MJ, Gafni A, Birch S. Portfolio theory and the alternative decision rule of cost-effectiveness analysis: theoretical and practical considerations. Soc Sci Med. 2004 May;58(10):1853-5. https://doi.org/10.1016/i.socscimed.2004.01.001

29. Braga MM, Floriano I, Mendes FM, Gafni A, Birch S. Implementing early caries detection and management for children: Analyzing the uncertainty of the effects and costs after one year of follow-up. EuHEA Conference 2018. Maastricht: EuHEA; 2018. 